\title{
Feasibility and Safety of a Novel Leg Exercise Apparatus for Venous Thromboembolism Prophylaxis After Total Joint Arthroplasty of the Lower Extremities
}

\section{Kenta Tanaka}

University of Tsukuba Faculty of Medicine: Tsukuba Daigaku Igaku Iryokei

Yukiyo Shimizu ( $\nabla$ shimiyukig@md.tsukuba.ac.jp)

University of Tsukuba https://orcid.org/0000-0001-7491-4516

\section{Hiroshi Kamada}

University of Tsukuba Faculty of Medicine: Tsukuba Daigaku Igaku Iryokei

\section{Shizu Aikawa}

Tsukuba Medical Center, Department of Cardiovascular surgery

\section{Hajime Mishima}

University of Tsukuba Faculty of Medicine: Tsukuba Daigaku Igaku Iryokei

\section{Akihiro Kanamori}

University of Tsukuba Faculty of Medicine: Tsukuba Daigaku Igaku Iryokei

\section{Tomofumi Nishino}

University of Tsukuba Faculty of Medicine: Tsukuba Daigaku Igaku Iryokei

\section{Masataka Sakane}

Tsukuba Gakuen Hospital, Depart of Orthopaedic Surgery

\section{Naoyuki Ochiai}

Kikkoman General Hospital, Department of Orthopaedic Surgery

\section{Masashi Yamazaki}

University of Tsukuba Faculty of Medicine: Tsukuba Daigaku Igaku Iryokei

\section{Research Article}

Keywords: Leg Exercise apparatus (LEX), venous thromboembolism prophylaxis, mechanical prophylaxis, arthroplasty, lower extremities

Posted Date: March 15th, 2021

DOI: https://doi.org/10.21203/rs.3.rs-293014/v1 
License: (c) (i) This work is licensed under a Creative Commons Attribution 4.0 International License. Read Full License 


\section{Abstract}

Background: Venous thromboembolism (VTE) is a severe complication of orthopedic surgeries. Although mechanical measures such as graduated compression stockings and intermittent pneumatic compression are widely used for VTE prevention, complications (e.g. lower limb pain or skin disorders) may result from prolonged usage. Early postoperative ambulation and active ankle movements are recommended but have low patient acceptance due to surgical pain and the lack of motivation. To overcome these limitations, we developed a novel leg exercise apparatus (LEX) to encourage limb movement in bedridden patients in the early postoperative period in order to prevent VTE. Here, we aimed to evaluate the feasibility and safety of the LEX in individuals at high risk of deep vein thrombosis (DVT) postoperatively.

Methods: Twenty subjects (4 men, 16 women) who underwent total joint arthroplasty in the lower extremity were enrolled in this prospective study. Exercise using LEX was performed for 5 minutes at $30 \mathrm{cycles} / \mathrm{min}$, four times per day from postoperative days 1 to 7 . Clinical assessments included the evaluation of vital signs before and after exercise with LEX, venous ultrasonography and blood tests within 7 days postoperatively, and evaluation of adverse events, including pulmonary embolism and cerebral hemorrhage.

Results: Overall, 16/20 (80\%) patients completed the exercise regimen of 7 days, while 4 dropped out. All four subjects who dropped out had undergone total hip arthroplasty; three of them refused to start exercises with LEX because of leg pain related to surgery. No severe adverse events occurred. There were no severe changes in vital signs. No DVT of the lower extremities was confirmed during postoperative week 1 . The average preoperative D-dimer level was $0.85 \mu \mathrm{g} / \mathrm{mL}$ (range, 0.4-2.2), whereas the corresponding value in postoperative week 1 was $7.93 \mu \mathrm{g} / \mathrm{mL}$ (range, 2.0-13.8).

Conclusions: In this study, no DVT cases or severe adverse events occurred postoperatively in patients undergoing total joint arthroplasty of the lower extremities who performed exercises with LEX. A study protocol analyzing the efficacy of this tool to prevent VTE after joint arthroplasty of the lower extremities may be safe and feasible.

\section{Background}

Venous thromboembolism (VTE) is a medical condition that includes deep vein thrombosis (DVT) and pulmonary embolism (PE) [1, 2]. Orthopedic surgery of the lower limbs constitutes a high-risk factor for VTE: patients undergoing total hip and knee arthroplasty show an incidence of this complication of approximately $0.6-1.5 \%[3,4]$. DVT of the lower limbs usually develops in the calf veins, especially those in the soleus muscle, and is a frequent source of pulmonary emboli [5-7].

The guidelines of the American College of Chest Physicians (ACCP) [8] and American Academy of Orthopaedic Surgeons (AAOS) [9] recommend mechanical and pharmacological thromboprophylaxis in patients undergoing orthopedic surgery. Specific measures to prevent lower extremity VTE include mechanical graduated compression stockings (GCS), intermittent pneumatic compression (IPC), and early ambulation, in addition to the administration of antithrombotic agents. Mechanical prophylaxis aims to avoid blood flow stasis, which is considered the main risk factor for DVT in "Virchow's triad" [10, 11]. IPC, 
which involves applying pressure on the foot and crus, has proven effective for the prevention of DVT in many clinical trials [12-14]. Additionally, voluntary lower extremity movements, such as those involved in early ambulation and active ankle exercising, are recommended based on their strong benefit on blood flow $[5,8.15]$. In this regard, using ultrasonography, Sochart and Hardinge [16] evaluated the venous blood flow during active ankle exercises and found a significant increase in volume and speed during automatic exercises, compared to bed rest or externally assisted exercises. Moreover, exercises involving ankle plantarflexion and dorsiflexion combined with varus-valgus angulation have a stronger effect on blood flow, compared to simple ankle plantar flexion or dorsiflexion exercises [16].

However, postoperative patients rarely perform lower extremity exercises voluntarily, even after being encouraged to do so; therefore, GCS and IPC are generally used as perioperative measures for mechanical VTE prophylaxis [12]. Many studies have confirmed the effectiveness of GCS and IPC in the prevention of thrombosis, but complications, such as lower limb pain or skin disorders, may result from prolonged usage [17].

Therefore, we developed a novel leg exercise apparatus (LEX) to facilitate active leg movement during the early postoperative period [18-21]. In a previous study on healthy adults, we found that LEX exercising is more effective than IPC alone for improving blood flow [18]. Moreover, other studies of our authorship have shown that LEX exercising of the lower extremities is significantly more effective in activating muscles of the lower limbs and improving blood flow than performing in-bed exercises without an assisting device [19].

The purpose of this study was to evaluate the feasibility and safety of LEX for VTE prophylaxis after total joint arthroplasty of the lower limbs.

\section{Methods}

\section{Participants}

This prospective study included 20 subjects ( 4 men, 16 women) who underwent total joint arthroplasty of the lower extremities for the first time between November 2014 and January 2016 at the University of Tsukuba Hospital. The operations included 19 total hip arthroplasties (THAs) and 1 total knee arthroplasty (TKA) (Table 1). Exclusion criteria were as follows: 1) history of DVT in the lower extremity or current anticoagulant treatment; 2 ) history of cerebral aneurysm or dissecting aortic aneurysm (conditions which may be aggravated by an increase in blood pressure); 3) uncontrolled hypertension; 4) serious conditions (e.g., heart, liver, and/or kidney disease); 5) history of malignancy within 5 years before the day of consent or suspicion of current malignancy; 6) confirmed or suspected pregnancy. 
Table 1

Background of the subjects

\begin{tabular}{|c|c|c|c|c|c|c|c|c|c|}
\hline subject & Sex & Age & Height & Weight & BMI & Surgery & GCS & IPCD & $\begin{array}{l}\text { Pharmacological } \\
\text { Therapy }\end{array}$ \\
\hline 1 & Male & 48 & 161.0 & 77.4 & 29.9 & THA & + & $\begin{array}{l}\text { Refusal } \\
\text { by } \\
\text { patient }\end{array}$ & Edoxaban \\
\hline 2 & Female & 70 & 158.0 & 54.8 & 22.0 & THA & + & + & - \\
\hline 3 & Female & 56 & 153.0 & 45.6 & 19.5 & THA & + & + & - \\
\hline 4 & Female & 63 & 151.1 & 62.0 & 27.2 & TKA & + & + & Edoxaban \\
\hline 5 & Female & 43 & 156.0 & 66.0 & 27.1 & THA & + & + & - \\
\hline 6 & Female & 38 & 155.7 & 53.4 & 22.0 & THA & + & + & - \\
\hline 7 & Female & 61 & 151.8 & 56.2 & 24.4 & THA & + & + & - \\
\hline 8 & Male & 38 & 168.5 & 76.8 & 27.0 & THA & + & + & - \\
\hline 9 & Female & 69 & 145.0 & 52.8 & 25.1 & THA & + & + & - \\
\hline 10 & Female & 75 & 141.0 & 39.0 & 19.6 & THA & + & + & - \\
\hline 11 & Female & 59 & 148.1 & 50.2 & 22.9 & THA & + & + & - \\
\hline 12 & Female & 72 & 131.5 & 36.6 & 21.2 & THA & + & + & - \\
\hline 13 & Female & 68 & 155.7 & 53.0 & 21.9 & THA & + & + & - \\
\hline 14 & Female & 55 & 155.0 & 49.4 & 20.6 & THA & + & + & - \\
\hline 15 & Female & 80 & 146.3 & 45.0 & 21.0 & THA & + & + & - \\
\hline 16 & Female & 71 & 151.0 & 63.0 & 27.6 & THA & + & + & - \\
\hline 17 & Female & 69 & 148.6 & 62.8 & 28.4 & THA & + & + & - \\
\hline 18 & Male & 56 & 168.9 & 82.8 & 29.0 & THA & + & + & - \\
\hline 19 & Female & 53 & 158.4 & 50.8 & 20.2 & THA & + & + & - \\
\hline 20 & Male & 67 & 151.4 & 70.7 & 30.8 & THA & + & + & - \\
\hline
\end{tabular}

LEX

The LEX [18-21] is a novel device originally developed to perform in-bed lower extremity exercises in patients with impaired mobilization. The device has been designed so that exercises can be performed in a 
supine position without diminishing the positive effect of ankle plantar flexion and dorsiflexion on blood flow.

The LEX is equipped with two (left/right) pedals and a movement control mechanism. The movement control mechanism comprises the base, main shaft, arm, pedal adjuster, and hooks (Fig. 1). The base is placed on the bed on which the patient lies, and fixed to the backboard of the bed using the hooks. The main shaft is placed at the center of the base. A straight arm is placed orthogonally at the top end of the main shaft, and pedals are attached to both ends of this arm. Since the arm rotates with the main shaft as the center, if the pedal on one side is pressed, the pedal on the other side moves. The foot is fixed to the pedal using a leather sole. The pedal allows ankle movements up to $30^{\circ}$ dorsiflexion, $60^{\circ}$ plantarflexion, $30^{\circ}$ subtalar inversion, and $20^{\circ}$ eversion, as the arm rotates. No extra load is added. LEX does not inhibit ankle movement, since in healthy individuals, the normal ankle movement ranges are $20^{\circ}$ dorsiflexion, $45^{\circ}$ plantar flexion, $30^{\circ}$ varus, and $20^{\circ}$ valgus. Moreover, since there is no frictional resistance between the leg and the bed, the device enables a smooth exercise performance that engages the knee and hip joints. Arch supports are placed inside each sole, enabling pressure to be applied to the plantar venous plexus as the pedals are pressed (Fig. 1). We intended to maintain knee flexion position to allow contraction of the soleus, the most fruent site of DVT in the lower leg $[5,6]$, to be predominant over that of the gastrocnemius; usually, with the knee in the extension position, the gastrocnemius contracts while the ankle plantar flexor bends more than the soleus. Exercising with the LEX is shown in detail in Additional file 1.

Finally, based on the findings of Sochart and Hardinge [16], the design of the LEX included pedals that enable complex exercises involving subtalar eversion or inversion in addition to dorsiflexion and plantarflexion.

\section{Primary outcomes}

The primary aims of the study were to determine the feasibility and safety of performing exercises using the LEX. To this purpose, we measured the degree of pain and fatigue and the effect on circulatory dynamics related to the use of the LEX. Pain and fatigue were assessed using the visual analog scale (VAS) and Borg scale [22], respectively (Table 2). Pain was measured using a specialized scale, and fatigue was assessed using a questionnaire. Pulse rate and blood pressure were assessed before and after exercise, and blood oxygen saturation level $\left(\mathrm{SpO}_{2}\right)$ was monitored during exercise. 
Table 2

The Borg scale: a subjective evaluation of

the degree of fatigue.

\begin{tabular}{|ll|}
\hline Borg scale \\
\hline 6 & \\
\hline 7 & Very, very light \\
\hline 9 & Very light \\
\hline 10 & \\
\hline 11 & Fairly light \\
\hline 12 & \\
\hline 13 & Somewhat hard \\
\hline 14 & \\
\hline 15 & Hard \\
\hline 16 & \\
\hline 17 & Very hard \\
\hline 18 & \\
\hline 19 & Very, very hard \\
\hline 20 & \\
\hline
\end{tabular}

Safety was assessed by registering the adverse events related to the LEX. Adverse events were defined as any medically unfavorable event observed during the study period. Pulmonary embolism, cerebral hemorrhage, and other fatal complications were considered severe adverse events. The safety standard in the present study was set as the non-occurrence of such severe adverse events.

\section{Secondary outcome}

The secondary outcome was the occurrence of DVT assessed using ultrasonography of the lower extremities and blood tests including D-dimer levels, both performed within seven days after surgery.

\section{Intervention}

Exercise was performed using the LEX device placed as explained above; the body position was adjusted using a specialized leg support (Fig. 2) to attain a knee flexion angle of $30^{\circ}$ in the supine position. 
The pace was set at 30 times/minute. Exercise sessions lasted for 5 minutes, and were repeated 4 times per day; in this regard, the study by Yamashita et al. [23], which investigated assisted exercise involving dorsiflexion and plantarflexion of the foot in postoperative patients, was used as a reference. Exercises were performed from postoperative days 1 to 7 .

All patients participated in preoperative practice exercises using LEX until they were comfortable with the device, to ensure an appropriate postoperative use. A physician supervised the first postoperative exercise session and provided instructions to conduct the exercise correctly and avoid any inappropriate movements or contraindicated positions.

Compliance with exercise was confirmed through a counter included in the device that recorded the number of times the pedals were pressed.

\section{Perioperative management and assessment}

Assessments and exercises were conducted according to the procedure shown in Table 3. Edoxaban was used in two cases according to risk analysis of VTE. (One is high BMI and the other is a TKA patient.) Thromboprophylaxis including mechanical and pharmacological methods are shown in Table 1.

Table 3

Procedure characteristics

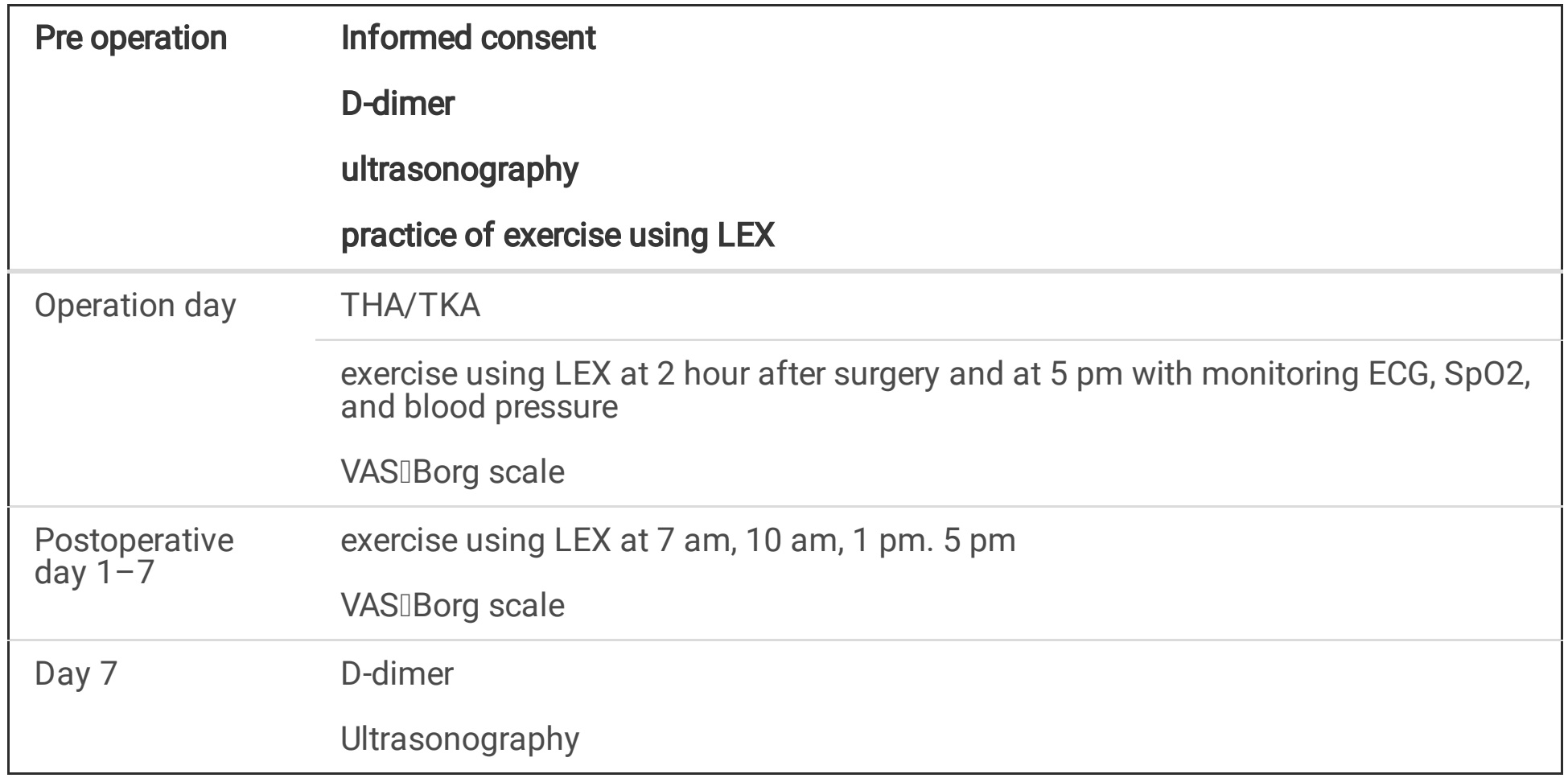

The IPC continuation was determined according to the performance in activities of daily living (ADLs), as assessed using the Barthel index (BI) [24] (Table 4). In patients who could mobilize in a wheelchair almost independently (BI: 5; movement ability: slight assistance required), IPC was used only at night, to avoid limiting ADLs. IPC use was stopped when the BI value reached 10 points (able to walk) and when the patient was able to walk approximately 45 meters using a walking assistance device. IPC was detached 
during exercise with the LEX. GCS were worn during the entire 7-day period. The risk of thromboembolism was assessed based on the Japanese Circulation Society Joint Working Group 2009 guidelines [5]. 
Table 4

The Barthel index for activities of daily living

\begin{tabular}{|l|}
\hline FEEDING \\
\hline $0=$ unable \\
\hline 5 = needs help cutting, spreading butter, etc., or requires modified diet \\
\hline BATHING \\
\hline $0=$ independent \\
\hline $5=$ independent (or in shower) \\
\hline GROOMING \\
\hline $0=$ needs to help with personal care \\
\hline $5=$ independent face/hair/teeth/shaving (implements provided) \\
\hline DRESSING \\
\hline $0=$ dependent \\
\hline $5=$ needs help but can do about half unaided \\
\hline $10=$ independent (including buttons, zips, laces, etc.) \\
\hline BOWELS \\
\hline $0=$ incontinent (or needs to be given enemas) \\
\hline $5=$ occasional accident \\
\hline $10=$ continent \\
\hline BLADDER \\
\hline $0=$ incontinent, or catheterized and unable to manage alone \\
\hline $5=$ occasional accident \\
\hline $10=$ continent \\
\hline TOILET USE \\
\hline $0=$ dependent \\
\hline $5=$ needs some help, but can do something alone \\
\hline $10=$ independent (on and off, dressing, wiping) \\
\hline TRANSFERS (BED TO CHAIR AND BACK) \\
\hline 0 unable, no sitting balance \\
\hline
\end{tabular}




\begin{tabular}{|l|}
\hline FEEDING \\
\hline 5 = major help (one or two people, physical), can sit \\
$10=$ minor help (verbal or physical) \\
$15=$ independent \\
\hline MOBILITY (ON LEVEL SURFACES) \\
0 = immobile or < 50 yards \\
\hline $5=$ wheelchair independent, including corners, $>50$ yards \\
\hline $10=$ walks with help of one person (verbal or physical) $>50$ yards \\
\hline $15=$ independent (but may use any aid; for example, stick) $>50$ yards \\
\hline STAIRS \\
\hline $0=$ unable \\
\hline $5=$ needs help (verbal, physical, carrying aid) \\
\hline $10=$ independent \\
\hline
\end{tabular}

\section{Cancellation criteria}

If any of the following events occurred during the study period, the patient was excluded and the specific reason and other relevant data were recorded in the case report: 1 ) adverse events that rendered continuation of the study difficult; 2 ) request from the patient to opt out of the study; 3 ) withdrawal of consent for participation (in this case, all data would be excluded); 4) confirmed critical or continued noncompliance to the study plan; and 5) other reasons the physician deemed sufficient for cancellation. The physician recorded all the content of the survey conducted until cancellation.

\section{Results}

Overall, 16 of 20 patients completed the exercise regimen of 7 days, while 4 dropped out. All four subjects who dropped out had undergone THA; three of them refused to initiate exercise due to leg pain related to surgery and the remaining one stopped exercising due to physical difficulties after 2 days. No severe adverse events occurred during the study period.

In the first exercise session (on the day of operation), the average VAS score was $38.5 \mathrm{~mm}$ (range, 0-85 $\mathrm{mm}$ ), and the average Borg scale score was 13 (range, 7-19).

Blood pressure was assessed in 15 patients. An increase in systolic blood pressure before or after exercise was observed in seven, while an increase in diastolic blood pressure before or after exercise was observed 
in six. The maximum difference between systolic blood pressure before and after exercise was $11 \mathrm{mmHg}$, whereas for diastolic blood pressure, the corresponding value was $19 \mathrm{mmHg}$ (Fig. 3).

Pulse rate was evaluated in 17 cases. The pulse rate increased in 14 subjects, with a maximum of 117 beats/min (bpm), and the maximum difference before and after exercise was 17 bpm (Fig. 4).

In some cases, a maximum reduction of $2 \%$ in $\mathrm{SpO}_{2}$ was observed, but there were no complaints of breathing difficulty (Fig. 5).

Regarding the secondary outcome, no DVT of the lower extremities was confirmed during postoperative week 1. The average preoperative D-dimer level was $0.85 \mu \mathrm{g} / \mathrm{mL}$ (range, 0.4-2.2), whereas the corresponding value in postoperative week 1 was $7.93 \mu \mathrm{g} / \mathrm{mL}$ (range, 2.0-13.8).

\section{Discussion}

In this study, the LEX was successfully used in patients after total joint arthroplasty of the lower extremities, and no severe adverse events occurred. Overall, $80 \%$ of subjects completed the 1-week protocol. This rate of acceptance is comparable to that of IPC, which is reported to be $81 \%[17,25]$.

With respect to the safety of a device that affects circulatory dynamics through exercise, none of the measured values for systolic or diastolic blood pressure, pulse rate, and $\mathrm{SpO}_{2}$ exceeded the exercise cancellation standard set by the Japanese Association of Rehabilitation Medicine [26] (Table 5). This suggests that it is unlikely that exercising with the LEX will cause any hemodynamic complication.

Table 5

Stop exercise criteria in this study.

\begin{tabular}{|lll|}
\hline & Stop exercise criteria & Present study \\
\hline Systolic blood pressure & Increasing $>40 \mathrm{mmHg}$ & Increasing $12 \mathrm{mmHg}$ \\
\hline Diastolic blood pressure & Increasing $>20 \mathrm{mmHg}$ & Increasing $19 \mathrm{mmHg}$ \\
\hline Pulse rate & Over $140 \mathrm{bpm}$ & Max 112bpm \\
\hline Sp02 & Moderate breathing difficulties & No breathing difficulties \\
\hline $\begin{array}{l}\text { Criteria are according to the guidelines for safety management and promotion in rehabilitation } \\
\text { medicine, proposed by the Japanese Association of Rehabilitation Medicine [26]. }\end{array}$ \\
\hline
\end{tabular}

According to the ACCP [8] and AAOS guidelines [9] the main aim of thromboprophylaxis is the prevention of lethal or symptomatic VTE; compared to previous guidelines, anticoagulant therapy indications are more restricted due to the risk of major bleeding complications and cost-effectiveness issues. Specifically, the AAOS guidelines clarify that pharmacological prophylaxis is only recommended for patients who are not at elevated risk of bleeding after surgery [27]. This guideline recommends mechanical prevention for asymptomatic VTE in patients with high risk of bleeding. 
Sashi et al. [28] studied the VTE rates in total hip and knee arthroplasty between 2002 and 2011 and found that, despite a slight decrease in VTE incidence related to both surgeries, PE rates remained stable. In detail, the overall median DVT and PE incidences in the cited study were, respectively, 0.40 and 0.23 in primary THA, and 0.62 and 0.34 in primary TKA. Chan et al. [29] conducted a systematic review of randomized controlled trials comparing the rates of VTE and bleeding due to pharmacological prophylaxis after THA or TKA and found an overall VTE rate of $0.99 \%$, which is similar to that of previous studies. However, the postoperative bleeding rate was $3.44 \%$, more than three times the VTE rate. Fuji et al. (4) studied that the development, prophylaxis, and treatment of VTE and bleeding events in 36,947 patients who had undergone orthopedic surgeries of the lower extremities from 2008 to 2013 using a healthcare database. They reported that the incidences of DVT, PE, and bleeding were $1.3 \%, 0.2 \%$, and $1.0 \%$ for TKA and $0.9 \%, 0.2 \%$, and $1.1 \%$ for THA. Therefore, the rate of bleeding complications due to antithrombotic therapy was higher than that of PE in both studies.

In turn, Tsuda et al. [30] reported the incidence of DVT using mechanical prophylaxis solely. Their study showed that among 184 cases of hip surgery receiving only IPC and GCS (patients with trauma were excluded), $5 \%$ were diagnosed with distal thromboses according to ultrasonic tests conducted in postoperative week 3 .

Considering the relatively high incidence of bleeding events, the current opinion is that individual risk analyses for VTE are needed [3,31]. In this regard, we are considering to design study protocols using LEX as mechanical prophylaxis according to VTE risk stratification, in addition or not to pharmacological prophylaxis.

Finally, in view of the current pandemic, it is worth noting that coronavirus disease 2019 (COVID-19) increases the risk of VTE significantly [32,33]. In addition to this, self-isolation periods prior to lower limb arthroplasty may increase the thrombotic risk further [31]. In a period in which close contact should be reduced to a minimum, mechanical devices which enable self-managed exercise, such as the LEX, may be valuable for both a safe DVT prophylaxis and an appropriate rehabilitation.

There were some limitations in this study. First, the sample size was too small to evaluate effectiveness in the prevention of VTE. However, considering that DVT after arthroplasty of the lower limbs is very common, it may be significant that no case of DVT was observed in our cohort. Second, we analyzed LEX use in patients who were independent in their ADLs before surgery and therefore could resume ambulation relatively soon after surgery. Third, patients in our study received other prophylactic methods such as IPC and anticoagulants, which can account for the absence of VTE cases. We are considering to evaluate the efficacy of the LEX in combination with established strategies to prevent VTE in bedridden patients.

\section{Conclusions}

In this study, the use of the LEX after total joint arthroplasty of the lower extremities was associated with a very good acceptance and no severe adverse events. Additionally, no cases of DVT were observed postoperatively. Our results suggest that a study protocol of lower extremity exercises using the LEX for

Page 13/21 
VTE prophylaxis in this group of patients is safe and feasible. We believe that LEX may be useful in patients with high VTE and bleeding risks, such as those with malignancies or undergoing spine surgery, in whom the use of pharmacological prophylaxis may be of concern. Additionally, the LEX has a potential educational value for patients and medical experts since it could be used to teach the importance of active leg exercises for thromboembolism prophylaxis.

\section{Abbreviations}

AAOS: American Academy of Orthopaedic Surgeons

ACCP: American College of Chest Physicians

ADLs: activities of daily living

Bl: Barthel index

COVID-19: coronavirus disease 2019

DVT: deep venous thrombosis

GCS: graduated compression stockings

IPC: intermittent pneumatic compression

LEX: leg exercise apparatus

PE: pulmonary embolism

Sp02: blood oxygen saturation level

THA: total hip arthroplasty

TKA: total knee arthroplasty

VAS: visual analog scale

VTE: venous thromboembolism

\section{Declarations}

\section{Ethics approval and consent to participate}

This study was approved by the Institutional Review Board of the University of Tsukuba Hospital, Japan (approval No. H17-18, Date of Registration is June 3, 2013). All patients provided consent for participation, both verbally and in written form, based on Ethics Committee recommendations. 
All patients provided consent for participation and publication of this study both verbally and in written form, based on Ethics Committee recommendations.

\section{Availability of data and materials}

All data generated or analyzed during this study are included in this article.

\section{Competing interests}

The authors declare that they have no competing interests.

\section{Funding}

This study was supported by the Ibaraki Global Niche Top Companies Incubation Project of Ibaraki Prefecture. The funding body had no role in the design of the study, collection, analysis, and interpretation of data, or writing of the manuscript.

\section{Author's contributions}

All authors participated in the design, execution, and analysis of this study, and read and approved the final version of the manuscript. KT, YS, and HK participated in the study design, drafted the manuscript, conducted the LEX interventions, and performed the data analysis. SA retrieved ultrasonographic data and helped draft the manuscript. HM, AK, and TN evaluated the participants clinically and helped draft the manuscript. MS and NO developed the device and participated in its design. MY was the principal investigator of this study, and participated in its design and coordination.

\section{Acknowledgments}

The authors thank Mr. Toshiji Mizukoshi, executive adviser of Nemoto, Co., Ltd., and Mr. Tsutomu Mizukoshi, the representative of Nemoto, Co., Ltd, as co-producers of the leg exercise apparatus. We would also like to thank the late Kiyoshi Eguchi, former professor of the University of Tsukuba Hospital, for his generous support and warm encouragement.

\section{References}

1. Ortel TL, Neumann I, Ageno W, Beyth R, Clark NP, Cuker A, et al. American Society of Hematology 2020 guidelines for management of venous thromboembolism: treatment of deep vein thrombosis and pulmonary embolism. Blood Adv. 2020;4(19):4693-738.

2. Lichota A, Szewczyk EM, Gwozdzinski K. Factors affecting the formation and treatment of thrombosis by natural and synthetic compounds. Int J Mol Sci. 2020;21(21):7975.

3. Santana DC, Emara AK, Orr MN, Klika AK, Higuera CA, Krebs VE, et al. An update on venous thromboembolism rates and prophylaxis in hip and knee arthroplasty in 2020. Medicina (Kaunas). 2020;56(9). 
4. Fuji T, Akagi M, Abe Y, Oda E, Matsubayashi D, Ota K, et al. Incidence of venous thromboembolism and bleeding events in patients with lower extremity orthopedic surgery: a retrospective analysis of a Japanese healthcare database. J Orthop Surg Res. 2017;12(1):55.

5. JCS Joint Working Group. Guidelines for the diagnosis, treatment and prevention of pulmonary thromboembolism and deep vein thrombosis (JCS 2009). Circ J. 2011;75(5):1258-81.

6. Ohgi S, Tachibana M, Ikebuchi M, Kanaoka Y, Maeda T, Mori T. Pulmonary embolism in patients with isolated soleal vein thrombosis. Angiology. 1998;49(9):759-64.

7. Kageyama N, Ro A, Tanifuji T, Fukunaga T. Significance of the soleal vein and its drainage veins in cases of massive pulmonary thromboembolism. Ann Vasc Dis. 2008;1(1):35-9.

8. Falck-Ytter Y, Francis CW, Johanson NA, Curley C, Dahl OE, Schulman S, et al. Prevention of VTE in orthopedic surgery patients: antithrombotic therapy and prevention of thrombosis, 9th ed: American College of Chest Physicians Evidence-Based Clinical Practice Guidelines. Chest. 2012;141(2 Suppl):e278S-325S.

9. Mont MA, Jacobs JJ, Boggio LN, Bozic KJ, Della Valle CJ, Goodman SB, et al. Preventing venous thromboembolic disease in patients undergoing elective hip and knee arthroplasty. J Am Acad Orthop Surg. 2011;19(12):768-76.

10. Malone PC, Agutter PS. The aetiology of deep venous thrombosis. QJM. 2006;99(9):581-93.

11. Brotman DJ, Deitcher SR, Lip GY, Matzdorff AC. Virchow's triad revisited. South Med J. 2004;97(2):2134.

12. Kakkos SK, Caprini JA, Geroulakos G, Nicolaides AN, Stansby GP, Reddy DJ. Combined intermittent pneumatic leg compression and pharmacological prophylaxis for prevention of venous thromboembolism in high-risk patients. Eur J Vasc Endovasc Surg. 2009;37(3):364-5.

13. Rohrer $\mathrm{O}$, Eicher M. Effectiveness of intermittent pneumatic compression (IPC) on thrombosis prophylaxis: a systematic literature review. Pflege. 2006;19(3):175.

14. Hanison E, Corbett K. Non-pharmacological interventions for the prevention of venous thromboembolism: a literature review. Nursing Stand. 1987. 2016;31(8):48.

15. Wickham N, Gallus AS, Walters BNJ, Wilson A, Ada NVPG, Committee NVPGA. Prevention of venous thromboembolism in patients admitted to Australian hospitals: summary of National Health and Medical Research Council clinical practice guideline. Int Med J. 2012;42(6):698-708.

16. Sochart $\mathrm{DH}$, Hardinge $\mathrm{K}$. The relationship of foot and ankle movements to venous return in the lower limb. J Bone Joint Surg Br. 1999;81(4):700-4.

17. Anand $S$, Asumu T. Patient acceptance of a foot pump device used for thromboprophylaxis. Acta Orthop Belg. 2007;73(3):386-9.

18. Shimizu Y, Kamada H, Sakane M, Aikawa S, Mutsuzaki H, Tanaka K, et al. A novel apparatus for active leg exercise improves venous flow in the lower extremity. J Sports Med Phys Fitness. 2016;56(12):1592-7.

19. Tanaka K, Kamada H, Shimizu Y, Aikawa S, Nishino T, Ochiai N, et al. The use of a novel in-bed active Leg Exercise Apparatus (LEX) for increasing venous blood flow. J Rural Med. 2016;11(1):11-6. 
20. Tanaka K, Kamada H, Shimizu Y, Aikawa S, Irie S, Ochiai N, et al. Muscle activity in the lower limbs during push-down movement with a new active-exercise apparatus for the leg. J Phys Ther Sci. 2016;28(3):1050-4.

21. Shimizu Y, Kamada H, Sakane M, Aikawa S, Mutsuzaki H, Tanaka K, et al. A novel exercise device for venous thromboembolism prophylaxis improves venous flow in bed versus ankle movement exercises in healthy volunteers. J Orthop Surg (Hong Kong). 2017;25(3):230949901773947.

22. Borg GA. Psychophysical bases of perceived exertion. Med Sci Sports Exerc. 1982;14(5):377-81.

23. Yamashita K, Yokoyama T, Kitaoka N, Nishiyama T, Manabe M. Blood flow velocity of the femoral vein with foot exercise compared to pneumatic foot compression. J Clin Anesth. 2005;17(2):102-5.

24. Mahoney FI, Barthel DW. FUNCTIONAL EVALUATION: THE BARTHEL INDEX. Md State Med J. 1965;14:61-5.

25. Dohm M, Williams KM, Novotny T. Micro-mobile foot compression device compared with pneumatic compression device. Clin Orthop Relat Res. 2011;469(6):1692-700.

26. Maeda M. Guideline for safety management and promotion in rehabilitation medicine. Jpn J Rehabil Med. 2007;44(7):384-90. (in Japanese)

27. Jacobs JJ, Mont MA, Bozic KJ, Della Valle CJ, Goodman SB, Lewis CG, et al. American Academy of Orthopaedic Surgeons clinical practice guideline on: preventing venous thromboembolic disease in patients undergoing elective hip and knee arthroplasty. J Bone Joint Surgery Am. 2012;94(8):746-7.

28. Shahi A, Chen AF, Tan TL, Maltenfort MG, Kucukdurmaz F, Parvizi J. The incidence and economic burden of in-hospital venous thromboembolism in the United States. J Arthroplasty. 2017;32(4):1063-6.

29. Chan NC, Siegal D, Lauw MN, Ginsberg JS, Eikelboom JW, Guyatt GH, et al. A systematic review of contemporary trials of anticoagulants in orthopaedic thromboprophylaxis: suggestions for a radical reappraisal. J Thromb Thrombolysis. 2015;40(2):231-9.

30. Tsuda K, Kawasaki T, Nakamura N, Yoshikawa H, Sugano N. Natural course of asymptomatic deep venous thrombosis in hip surgery without pharmacologic thromboprophylaxis in an Asian population. Clin Orthop Relat Res. 2010;468(9):2430-6.

31. Kahn SR, Shivakumar S. What's new in VTE risk and prevention in orthopedic surgery. Res Pract Thromb Haemost. 2020;4(3):366-76.

32. Khan SA, Logan P, Asokan A, Handford C, Rajgor HD, Khadabadi NA, et al. The incidence of venous thromboembolism in total joint replacement during COVID-19 pandemic: has lockdown had an influence? Bone Jt Open. 2020;1(12):751-6.

33. Cai C, Guo Y, You Y, Hu K, Cai F, Xie M, et al. Deep venous thrombosis in COVID-19 patients: a cohort analysis. Clin Appl Thromb Hemost. 2020;26:1076029620982669.

\section{Figures}



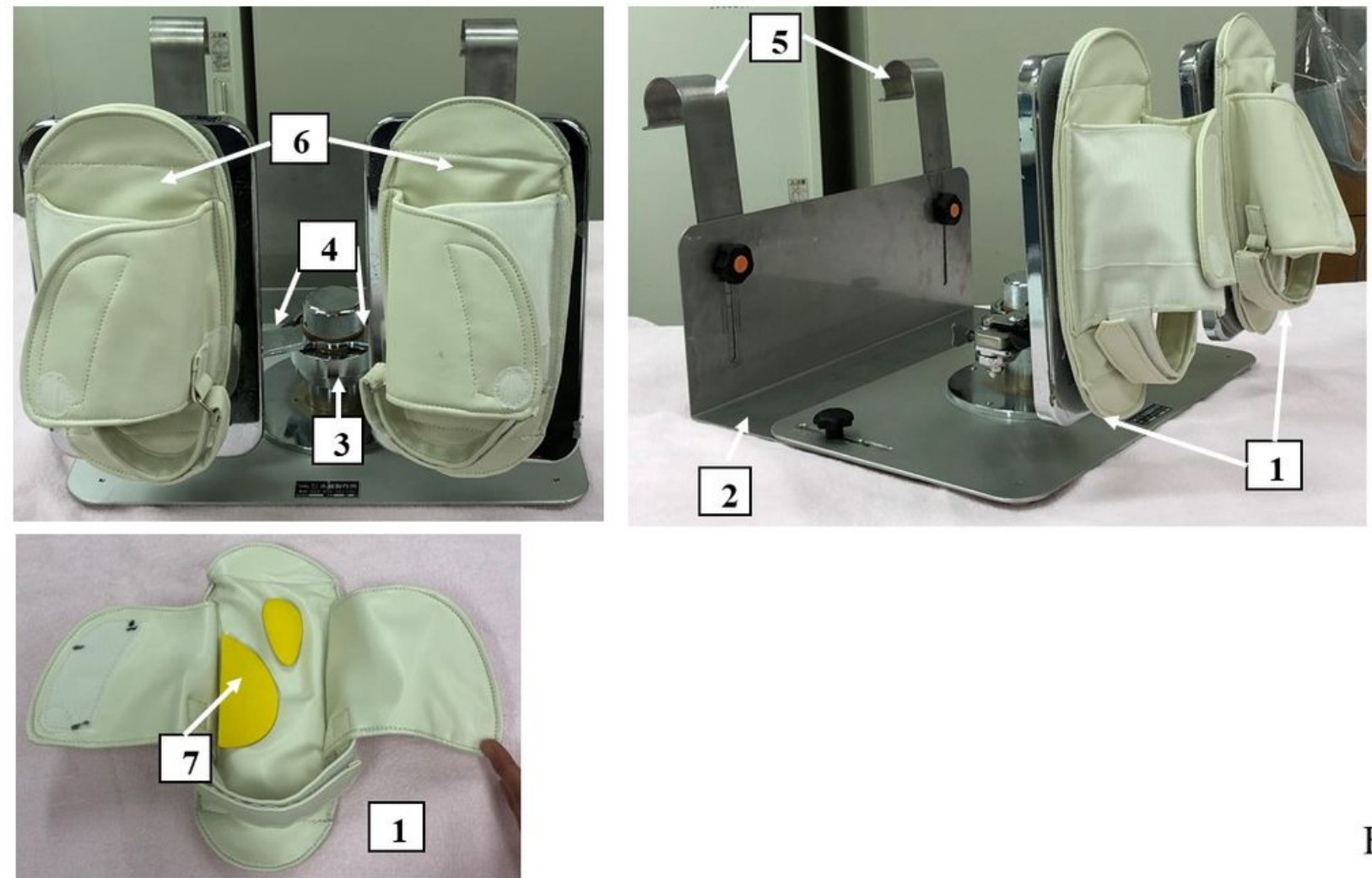

Fig. 1

Figure 1

Structure of the LEX. 1: pedals, 2: base, 3: main shaft, 4: arms, 5: hooks, 6: sole, 7: arch supports placed inside the sole

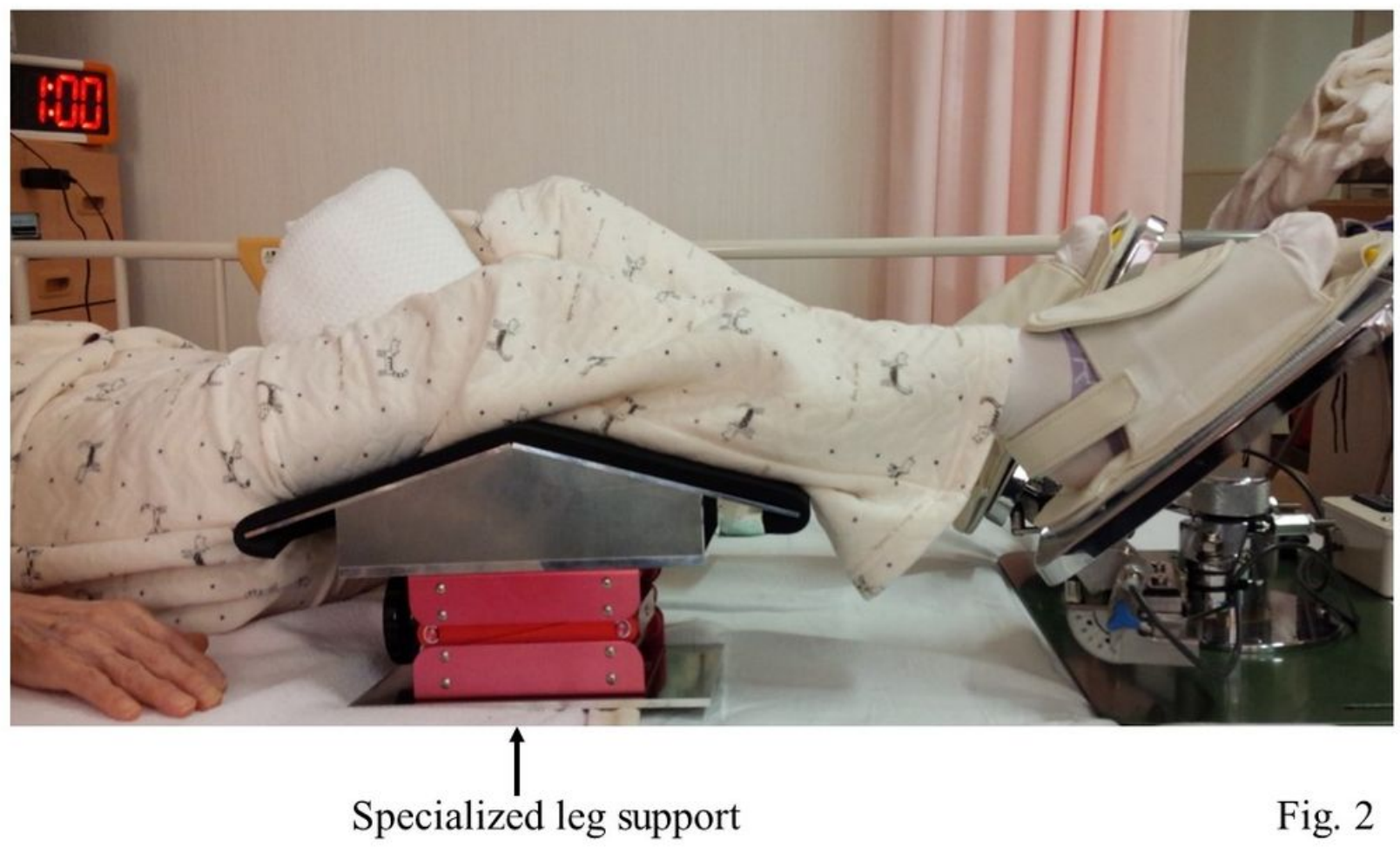


Figure 2

Exercise position using the LEX with a specialized leg support

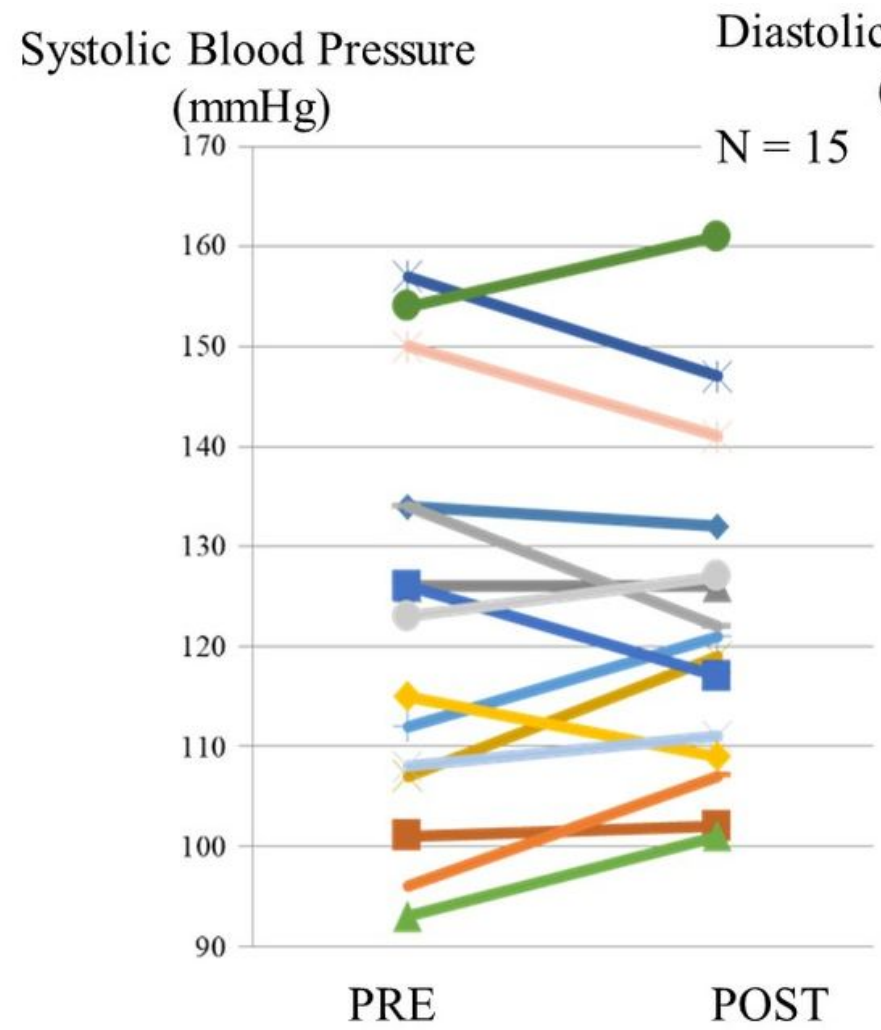
( $\mathrm{mmHg}$ )

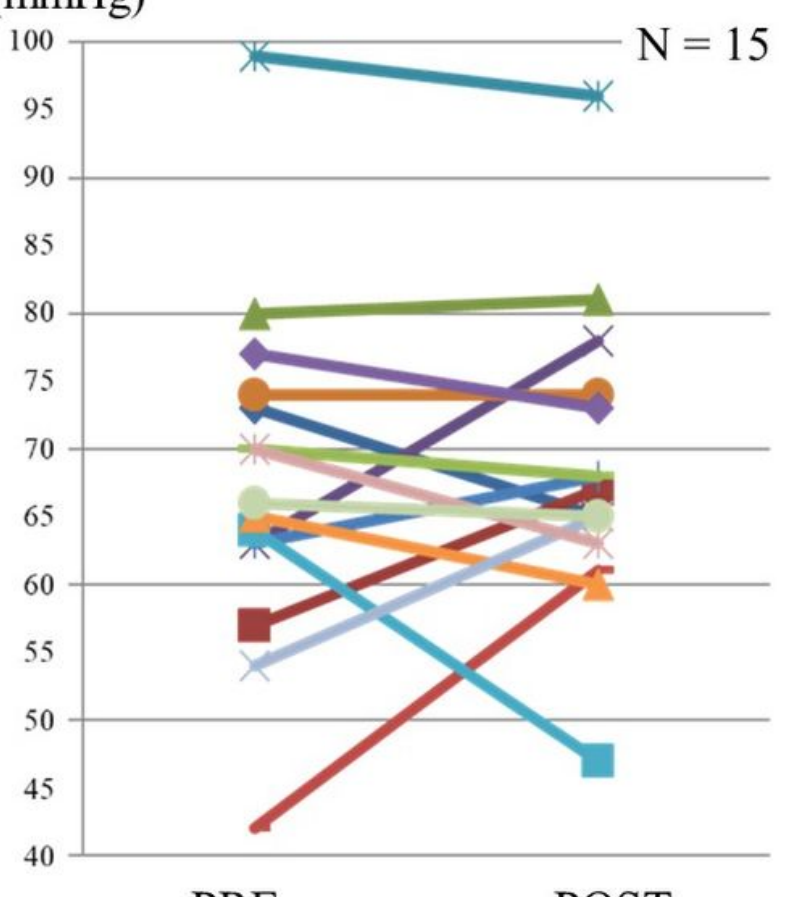

PRE

Fig. 3

\section{Figure 3}

Blood pressure before and after exercise using the LEX 


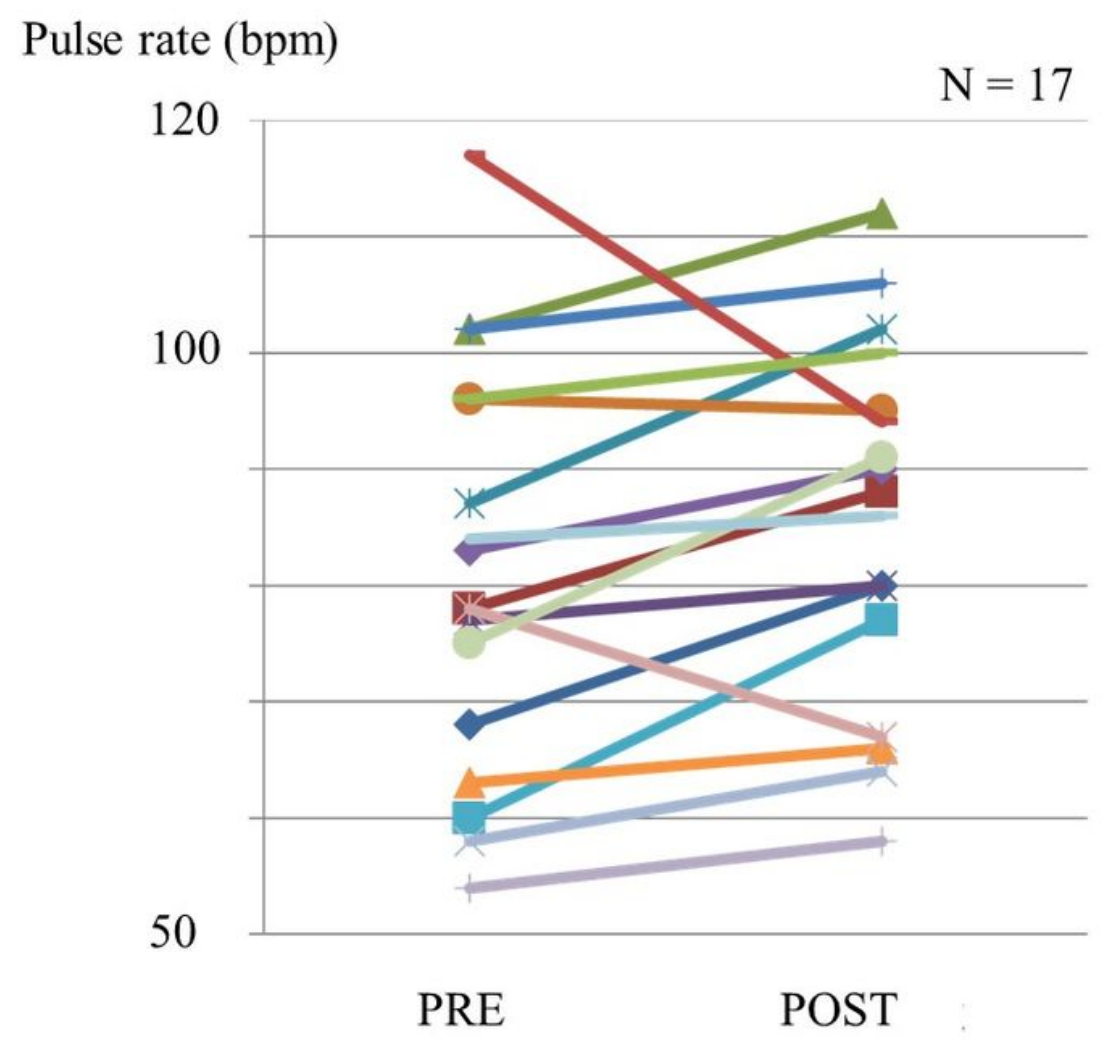

Fig. 4

Figure 4

Pulse rate before and after exercise using the LEX

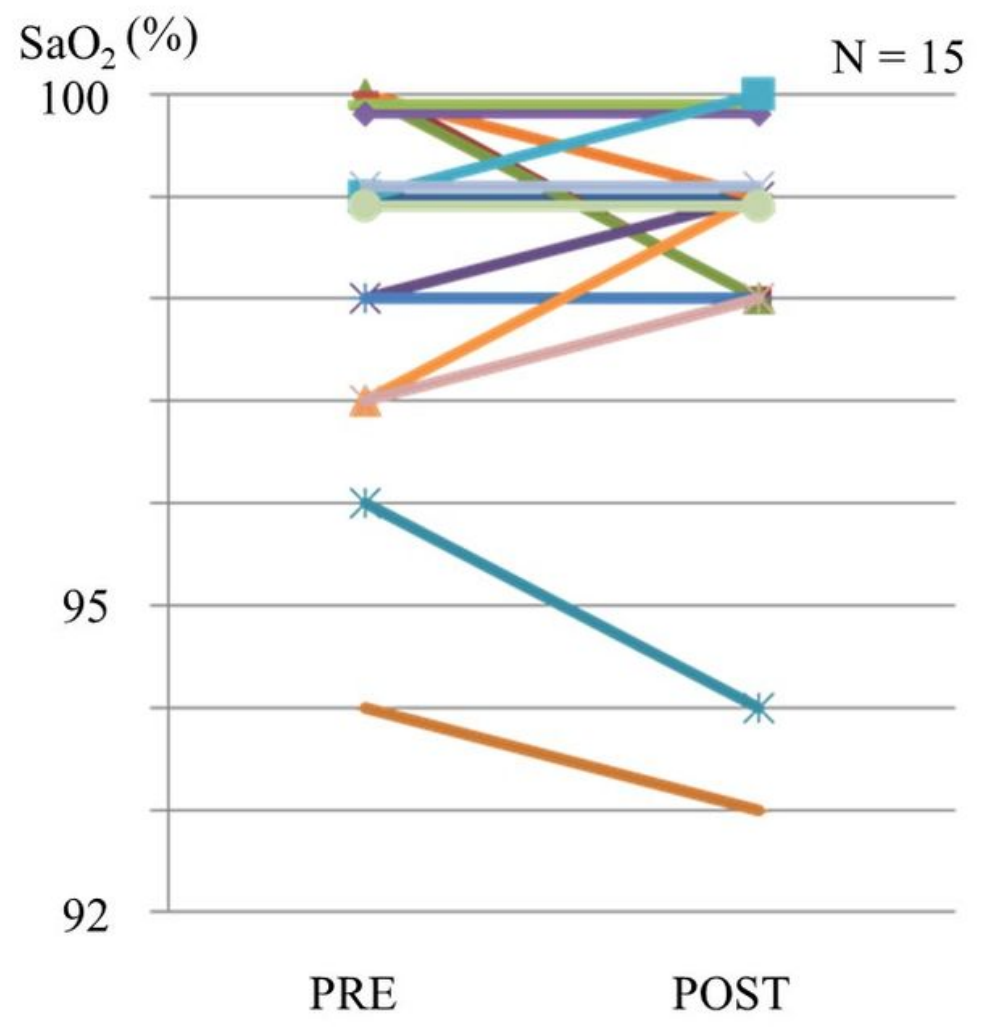

Fig. 5

Figure 5 
SpO2 before and after exercise using LEX

\section{Supplementary Files}

This is a list of supplementary files associated with this preprint. Click to download.

- Additionalfile1.mp4 